\title{
Risk Minimizing Strategies for Tracking a Stochastic Target $^{\star}$
}

\author{
Andrzej Palczewski \\ Faculty of Mathematics, University of Warsaw, Banacha 2, 02-097 Warszawa, Poland \\ A.Palczewski@mimuw.edu.pl
}

\begin{abstract}
We consider a stochastic control problem of beating a stochastic benchmark. The problem is considered in an incomplete market setting with external economic factors. The investor preferences are modelled in terms of HARA-type utility functions and trading takes place in a finite time horizon. The objective of the investor is to minimize his expected loss from the outperformance of the benchmark compared to the portfolio terminal wealth, and to specify the optimal investment strategy. We prove that for considered loss functions the corresponding Bellman equation possesses a unique solution. This solution guaranties the existence of a well defined investment strategy. We prove also under which conditions the verification theorem for the obtained solution of the Bellman equation holds.
\end{abstract}

Keywords: optimal portfolios, stochastic target, benchmark tracking.

\section{Introduction}

We analyze the optimal portfolio and investment policy for an investor who is concerned about his wealth relative to the performance of a given benchmark. The benchmark evolves stochastically over time and the investor's objective is to minimize his loss with respect to this benchmark by investing in a portfolio of stochastically evolving financial instruments. Since the benchmark is not necessarily perfectly correlated with the investment opportunities, we are in the framework of an incomplete market, and there is no investment policy under which the investor can outperform the benchmark with certainty.

The portfolio problem where the objective is to exceed the performance of a selected target benchmark is sometimes called an active portfolio management. It is well known that many professional investors apply this benchmarking procedure. However, many small investors follow a benchmarking procedure as well, by trying to beat inflation, exchange rates, or other market indices.

The problem of an investment portfolio which outperforms a given benchmark has been studied for a long time. For objectives such as maximizing the probability that the investor's wealth achieves a certain performance goal relative to the benchmark, before falling below to a predetermined shortfall, or minimizing the expected time to reach the performance goal, the problem is studied by Browne [4], [5]. For the special case

\footnotetext{
${ }^{\star}$ Financial support from MNiSzW grant no. NN-201-547838 is gratefully acknowledged.
}

D. Hömberg and F. Tröltzsch (Eds.): CSMO 2011, IFIP AICT 391, pp. 188-196, 2013.

(C) IFIP International Federation for Information Processing 2013 
where the benchmark is perfectly correlated with the investment opportunities, these problems over a finite-horizon are analyzed in [4], and for a more general model that the benchmark is not perfectly correlated with the investment opportunity in [5]. The problem of finding the minimal initial data of a controlled process which guarantees to reach the benchmark with a given probability of success or, more generally, with a given level of expected loss was first introduced by Föllmer and Leukert [7] in the context of quantile hedging. This approach has been then extended to the stochastic target problem studied by Soner and Touzi [9, 10], and in a number of papers by Bouchard et. al. [1, 2, 3].

In opposition to the majority of previously mentioned papers, in this paper, we study a loss minimization objective when the prices of financial instruments are functions of external economic factors. A similar problem but without taking into account economic factors is solved by Browne [5]. The absence of economic factors makes the problem much simpler as the HJB equation is reduced in that case to an ODE. In the presence of external factors the HJB equation becomes a multidimensional nonlinear PDE for which the existence of solutions is a challenging problem. We solve this problem using the well developed theory of quasilinear parabolic equations. We also show that under suitable regularity assumptions the verification theorem holds. Hence, the obtained solution to the HJB equation is a solution to the optimization problem. The plan of the paper is as follows. In Section 2, we present the portfolio problem arising from the active portfolio management. In Section 3, we show that, under additional assumptions on the loss function, we can find a smooth solution to the HJB equation and construct effectively an optimal investment strategy. Section 4 is devoted to the formulation and proof of the verification theorem.

\section{The Portfolio Problem}

We consider the portfolio problem in which the prices of securities are functions of external state variables (economic factors). Our goal is to construct a portfolio which can outperform a stochastic benchmark. We consider a general setting of the problem. In particular, the risk factors which define the dynamics of the benchmark can be different from the risk factors in the dynamics of securities. Hence, the problem is an incomplete market problem.

The setting of the market is as follows: we have a market defined on a probability space $(\Omega, \mathcal{F}, \mathbb{P})$ with the filtration $\left(\mathcal{F}_{t}\right)_{t \in[0, T]}$ generated by $d$-dimensional standard Wiener process $W(t)=\left(W_{1}, \ldots, W_{d}\right)$ (in what follows we treat $W$ as a column vector). On that probability space we have $N$ stochastic processes describing the prices of securities with the dynamics

$$
\frac{d S_{i}(t)}{S_{i}(t)}=\mu_{i}(t, R) d t+\sum_{j=1}^{d} \sigma_{i j}(t, R) d W_{j}(t), \quad i=1,2, \ldots, N
$$

where $\mu_{i}$ and $\sigma_{i j}$ depend on an $M$-dimensional vector of economic factors $R$. 
We assume that the dynamics of factors $R$ follow the Markovian diffusion process

$$
d R_{m}(t)=\mu_{m}^{r}(t, R) d t+\sum_{i=1}^{d} b_{m i}(t, R) d W_{i}(t), \quad m=1,2, \ldots, M .
$$

It is convenient to switch to vector notation and introduce the matrices $\sigma=\left(\sigma_{i j}\right)$, $B=\left(b_{i j}\right)$ and the column vectors $\mu=\left(\mu_{1}, \ldots, \mu_{N}\right)^{\prime}, \mu^{r}=\left(\mu_{1}^{r}, \ldots, \mu_{M}^{r}\right)^{\prime}$. (Here and in what follows $x^{\prime}$ denotes the transpose of the matrix or vector $x$.)

Assumption 2.1. For the model of security prices we assume that coefficients $\mu(t, r)$ and $\sigma(t, r)$ are deterministic functions bounded and continuous for $t \in[0, T]$ and $r \in$ $\mathbb{R}^{M}$. For the model of economic factors we make typical assumptions which guarantee the existence of strong solutions to equation (2), i.e., we assume that $\mu^{r}(t, r)$ and $B(t, r)$ are deterministic continuous functions of their arguments, which in addition fulfil the estimates

$$
\begin{aligned}
& \left\|\mu^{r}\left(t, r_{1}\right)-\mu^{r}\left(t, r_{2}\right)\right\|+\left\|B\left(t, r_{1}\right)-B\left(t, r_{2}\right)\right\| \leq c\left\|r_{1}-r_{2}\right\| \\
& \left\|\mu^{r}(t, r)\right\|^{2}+\|B(t, r)\|^{2} \leq c^{2}\left(1+\|r\|^{2}\right)
\end{aligned}
$$

for $t \in[0, T], r, r_{1}, r_{2} \in \mathbb{R}^{M}$, where $c$ is a positive constant.

We analyze the stochastic target problem in an incomplete market assuming that the dimension of risk factors is high, i.e. dimmension $d$ of the Wiener process $W$ is high, and the number of securities and economic factors much lower. This means that $d \gg N$ and $d \gg M$. To guarantee well-posedness and solvability of the optimization problem, we have to make additional assumptions.

Assumption 2.2. About the model of securities dynamics we assume a "partial invertibility" of the model, i.e., the matrix $\Sigma=\sigma \sigma^{\prime}$ is nonsingular and $\Sigma^{-1}(t, r)$ is bounded for $t \in[0, T]$ and $r \in \mathbb{R}^{M}$. This in fact means that securities are driven by $N$ risk factors and limited to these $N$ dimensions the security market is complete.

About the model of dynamics of economic factors we assume that the matrix $B B^{\prime}$ is positive definite. Strictly speaking, we postulate that there exist positive constants $\nu_{1}$, $\nu_{2}$ such that for any $x \in \mathbb{R}^{M}$

$$
0<\nu_{1}\|x\|^{2} \leq x^{\prime} B B^{\prime} x \leq \nu_{2}\|x\|^{2} .
$$

The stochastic benchmark is modelled as a general log-normal stochastic process $H(t)$ which fulfils the equation

$$
d H(t):=H(t)\left(\mu^{H} d t+\xi d W(t)\right),
$$

where $\xi=\left(\xi_{1}, \ldots, \xi_{d}\right)^{\prime}$ is a column vector, and coefficients $\mu^{H}$ and $\xi$ are deterministic functions of $t \in[0, T]$ and $r \in \mathbb{R}^{M}$.

We consider now a portfolio $V(t)$ consisting of assets $S_{i}(t), i=1, \ldots, N$. Denoting by $\pi_{i}$ the fraction of the total wealth $V(t)$ invested in the security $S_{i}$, we can write

$$
d V^{\pi}(t)=V^{\pi}(t)\left(\mu^{V} d t+\theta^{V} d W(t)\right)
$$


where after introducing the column vector $\pi=\left(\pi_{1}, \ldots, \pi_{N}\right)^{\prime}$ we have $\mu^{V}=\mu^{\prime} \pi$ and $\theta^{V}=\sigma^{\prime} \pi$.

We define now the new process

$$
X(t)=\frac{H(t)}{V^{\pi}(t)} .
$$

This approach enables us to consider in the same framework losses and gains. Such an approach is not quite new in the financial literature. It is applied by Browne [4, 5]. A similar quotient is used by Dai Pra, Runggaldier and Tolotti [6] who optimize the quadratic loss in the benchmark tracking problem.

For the process $X(t)$ we obtain the following equation of evolution

$$
\frac{d X}{X}=\mu^{X} d t+\theta d W
$$

where

$$
\theta=\xi-\theta^{V}, \quad \mu^{X}=\mu^{H}-\mu^{V}-\theta^{\prime} \theta^{V} .
$$

We optimize the process $X(t)$ with respect to the vector of strategies $\pi$. Admissible strategies for our problem are defined as follows.

Definition 2.1. Let $U$ be a complete, separable metric space and $0<T<\infty$. We define the set of admissible strategies $\Pi(t, x, r)$ as fulfilling the conditions:

1. $\pi:[t, T] \times \Omega \rightarrow U \subseteq \mathbb{R}^{N}$ is measurable, bounded and $\left\{\mathcal{F}_{\tau}\right\}_{\tau \geq t}$-adapted, for each $\pi \in \Pi(t, x, r)$,

2. $X(t)=x$ (budget constraint),

3. $R(t)=r$.

We consider the optimization problem in the framework of utility theory. This means that we fix a utility function $g$ and optimize the terminal value of process $X$ measured by $g$. The optimization problem is of the form

$$
\min _{\pi \in \Pi(t, x, r)} \mathbb{E}[g(X(T)) \mid X(t)=x, R(t)=r] .
$$

In fact, it is better to call $g$ a loss function as our goal is to minimize losses and not to maximize gains.

Under Assumptions 2.1, 2.2 and Definition 2.1, equation (7) admits the unique solution and the value function

$$
u(t, x, r):=\min _{\pi \in \Pi(t, x, r)} \mathbb{E}[g(X(T)) \mid X(t)=x, R(t)=r]
$$

is well defined.

With this value function we arrive at the following Hamilton-Jacobi-Bellman equation

$$
\begin{aligned}
\partial_{t} u+\inf _{\pi \in \Pi}( & \mu^{X} x \partial_{x} u+\left(\mu^{r}\right)^{\prime} \nabla_{r} u+\frac{1}{2} \theta^{\prime} \theta x^{2} \partial_{x x} u+ \\
& \left.+\frac{1}{2} B B^{\prime}\left(\nabla_{r} \otimes \nabla_{r} u\right)+x \theta^{\prime} B^{\prime} \nabla_{r} \partial_{x} u\right)=0, \\
u(T, x, r)= & g(x),
\end{aligned}
$$


where $\nabla_{r}$ denotes the gradient operator with respect to vector variable $r$ (a column vector), $\partial_{t}$ and $\partial_{x}$ denote differential operator with respect to scalar variables $t$ and $x$, respectively. $\nabla_{r} \otimes \nabla_{r}$ has the following meaning: when $x$ and $y$ are $n$-dimensional column vectors then $x \otimes y$ denotes the $n \times n$ matrix $x y^{\prime}$, i.e. $\nabla_{r} \otimes \nabla_{r} u$ is a matrix of all second order derivatives of $u$ with respect to variables $r_{i}$ and $r_{j}, i, j=1, \ldots, M$.

\section{Smooth Solutions of the HJB Equation}

To obtain smooth solutions to the HJB equation (10), we make the following assumption.

Assumption 3.1. The loss function $g(x)$ is from the generalized HARA class and is given by the expression $g(x)=c x^{\alpha}$, for $\alpha>1$ and $x \in[0, \infty)$.

Remark 3.1. In fact, from the technical point of view, we can assume only that $g$ is such that $\alpha \neq-1$. The assumption $\alpha>1$ is essential when we want to interpret $g(x)$ as a loss function.

Under the above assumption, we postulate that the value function can be factorized in the form

$$
u(t, x, r)=g(x) q(t, r) .
$$

Substituting the above factorization into equation (10), we obtain the following PDE problem for $q$ :

$$
\begin{aligned}
& \partial_{t} q+\inf _{\pi \in \Pi}\left(\alpha \mu^{X} q+\left(\mu^{r}\right)^{\prime} \nabla_{r} q+\frac{\alpha(\alpha-1)}{2} \theta^{\prime} \theta q+\right. \\
& \left.+\frac{1}{2} B B^{\prime}\left(\nabla_{r} \otimes \nabla_{r} q\right)+\alpha \theta^{\prime} B^{\prime} \nabla_{r} q\right)=0 \\
& q(T, r)=1 .
\end{aligned}
$$

From equation (12) we can derive formally the optimal investment strategy

$$
\pi^{*}=\pi^{0}+\frac{\pi^{1} \nabla_{r} q}{q},
$$

where

$$
\begin{aligned}
\pi^{0} & =\frac{1}{1+\alpha} \Sigma^{-1}(\mu+\alpha \sigma \xi), \\
\pi^{1} & =\frac{1}{1+\alpha} \Sigma^{-1} \sigma B^{\prime} .
\end{aligned}
$$

Substituting expression (13) into the HJB equation (12) we obtain

$$
\begin{aligned}
\partial_{t} q & +\alpha \mu^{*} q+\left(\mu^{r}\right)^{\prime} \nabla_{r} q+\frac{\alpha(\alpha-1)}{2}\left(\theta^{*}\right)^{\prime} \theta^{*} q+ \\
& +\frac{1}{2} B B^{\prime}\left(\nabla_{r} \otimes \nabla_{r} q\right)+\alpha\left(\theta^{*}\right)^{\prime} B^{\prime} \nabla_{r} q=0 .
\end{aligned}
$$


In this equation, $\mu^{*}$ denotes the value of $\mu^{X}$, and $\theta^{*}$ the value of $\theta$ evaluated at the point of the optimal strategy $\pi^{*}$.

After rearrangements, the HJB equation (14) takes the form

$$
\partial_{t} q+A_{0}\left(\nabla_{r} \otimes \nabla_{r} q\right)+A_{1} \frac{\nabla_{r} q \otimes \nabla_{r} q}{q}+A_{2} \nabla_{r} q+A_{3} q=0
$$

where

$$
\begin{aligned}
& A_{0}=\frac{1}{2} B B^{\prime}, \\
& A_{1}=\frac{1}{2} \alpha(\alpha+1)\left(\pi^{1}\right)^{\prime} \Sigma \pi^{1}-\alpha B \sigma^{\prime} \pi^{1}, \\
& A_{2}=\mu^{r}+\alpha(\alpha+1)\left(\pi^{1}\right)^{\prime} \Sigma \pi^{0}-\alpha^{2}\left(\pi^{1}\right)^{\prime} \sigma \xi+\alpha\left(B \xi-\left(\pi^{1}\right)^{\prime} \mu-B \sigma^{\prime} \pi^{0}\right), \\
& A_{3}=\frac{1}{2} \alpha^{2}\left(\left(\pi^{0}\right)^{\prime} \Sigma \pi^{0}-2\left(\pi^{0}\right)^{\prime} \sigma \xi+\xi^{\prime} \xi\right)+\alpha\left(\mu^{H}-\mu^{\prime} \pi^{0}-\frac{1}{2} \xi^{\prime} \xi\right) .
\end{aligned}
$$

Equation (15) has to be solved in the strip $0 \leq t \leq T$ with the terminal condition

$$
q(T, r)=1 \text {. }
$$

Equation (15) is a quasilinear parabolic equation which possesses a solution provided this solution is bounded away from zero. To find this solution we make the substitution

$$
z=\ln q
$$

For the new function $z$ we obtain the equation

$$
\partial_{t} z+A_{0}\left(\nabla_{r} \otimes \nabla_{r} z\right)+\left(A_{0}+A_{1}\right) \nabla_{r} z \otimes \nabla_{r} z+A_{2} \nabla_{r} z+A_{3}=0,
$$

with the terminal condition

$$
z(T, r)=0 .
$$

To solve equation (17) with condition (18), we use well known results in the theory of quasilinear parabolic equations.

Let us consider a boundary value problem for a $n$-dimensional quasilinear parabolic equation

$$
\begin{aligned}
& \partial_{t} w-\sum_{i, j=1}^{n} a_{i j}(t, x) \partial_{x_{i} x_{j}} w+a\left(t, x, w, \partial_{x} w\right)=0, \quad \text { for }(t, x) \in \mathcal{O}_{T}, \\
& w(t, x)=\psi(t, x), \quad \text { for }(t, x) \in \Gamma_{T},
\end{aligned}
$$

in a bounded domain $\mathcal{O}_{T}=[0, T] \times \mathcal{O}$, where $\mathcal{O}$ is a bounded domain in $\mathbb{R}^{n}$ with the boundary of class $H^{2+\beta}$, and $\Gamma_{T}=\partial \mathcal{O} \times[0, T] \cup \mathcal{O} \times\{t=0\}$.

Theorem 7.4 in Chapter 6 of the book by Ladyzhenskaya, Solonnikov and Uraltseva [8] guarantees that, under suitable assumptions on coefficients $a_{i j}, a$ and boundary function $\psi$, there exists a unique solution to the boundary value problem (19) in $H^{1+\beta / 2,2+\beta}\left(\mathcal{O}_{T}\right)$.

To apply the above result to equation (17), we have to make additional assumptions. 
Assumption 3.2. Let functions $\mu, \mu^{r}, \mu^{H}, \sigma, B, \xi$ and $\Sigma^{-1}$ be Hölder continuous functions of $t$ with the Hölder exponent $\beta / 2$, and Hölder continuous functions of $r$ with the Hölder exponent $\beta$, for some $\beta>0$.

Now we can prove our main theorem.

Theorem 3.1. Under Assumptions 2.1, 2.2 3.1 3.2 and the assumptions of Definition 2.1. there exists a unique solution $z(t, r)$ to the terminal problem (17)-(18) and $|z|$, $\left|\partial_{t} z\right|,\left|\partial_{x_{i}} z\right|,\left|\partial_{x_{i} x_{j}} z\right|$ are bounded in $[0, T] \times \mathbb{R}^{M}$. This solution belongs to $H^{1+\beta / 2,2+\beta}\left(\mathcal{O}_{T}\right)$, where $\mathcal{O}_{T}=[0, T] \times \mathcal{O}$ and $\mathcal{O}$ is a bounded domain in $\mathbb{R}^{M}$.

Proof: Let us consider the terminal problem (17)-(18) in $\mathcal{O}_{T}=[0, T] \times \mathcal{O}$, where $\mathcal{O}$ is a fixed bounded domain in $\mathbb{R}^{M}$. To solve this problem, we supplement equation (17) and terminal condition (18) with the boundary condition

$$
z(t, r)=0 \quad \text { for }(t, r) \in \partial \mathcal{O} \times[0, T] .
$$

The above defined augmented problem fulfils already the assumptions of Theorem 7.4 in Chapter 6 of [8]. Due to this theorem, there exists a unique solution of equation (17) with terminal condition (18) and boundary condition (20). This solution together with its derivatives can be estimated in $\mathcal{O}_{T}$ with constants which depend only on constants present in the Assumptions, and not on the size of domain $\mathcal{O}$. Hence, the solution which exists in any bounded domain $\mathcal{O}_{T}$ belongs to $H^{1+\beta / 2,2+\beta}\left(\mathcal{O}_{T}\right)$ and is uniformly bounded together with its derivatives independently of the size of the domain. Then we can consider a increased sequence of bounded smooth domains $\mathcal{O}^{n}$ that fill in the whole $\mathbb{R}^{M}$ and solutions $z^{n}$ to problem (17), (18), (20) with $\mathcal{O}$ replaced by $\mathcal{O}^{n}$. By the standard Arzela-Ascoli theorem, we can choose a subsequence of $z^{n}$ which converges to a function which is a solution to $(17)-(18)$ on $[0, T] \times \mathbb{R}^{M}$.

Remark 3.2. In many situations, economic factors should be restricted to nonnegative values only. In that cases, Theorem 3.1 is still applicable as we can construct a sequence of bounded smooth domains $\mathcal{O}^{n}$ approximating the space $\mathbb{R}_{+}^{M}$.

Corollary 3.1. Let us observe that due to Theorem 3.1 fuction $z(t, r)$ and its derivatives are bounded. Returning back to the original function $q$, we conclude that $q(t, r)$ is bounded away from zero and the quotient $q_{r_{m}} / q$ is bounded. It follows than that the optimal investment strategy given by expression (13) is bounded and admissible in accordance with Definition 2.1.

\section{Verification Theorem}

Theorem 3.1 guaranties a smooth solution to the terminal problem (17)-(18). This solution is not necessarily a solution to the optimization problem. To prove the optimality, we need some additional results. First, we have to show that the solution to problem (17)-(18) is a function of class $C^{1,2}$ on $[0, T] \times \mathbb{R}^{M}$.

To this end, we can use Theorem 8.1 from Chapter 6 of [8] which guarantees the existence of a unique solution in $H^{1+\beta / 2,2+\beta}\left(Q_{T}\right)$, where $Q_{T}=[0, T] \times \mathbb{R}^{n}$, to the Cauchy problem for a quasilinear parabolic equation 


$$
\begin{aligned}
& \partial_{t} w-\sum_{i, j=1}^{n} a_{i j}(t, x) \partial_{x_{i} x_{j}} w+a\left(t, x, w, \partial_{x} w\right)=0, \text { in }(0, T] \times \mathbb{R}^{n}, \\
& w(0, x)=\psi(x), \text { in } \mathbb{R}^{n},
\end{aligned}
$$

if in addition to the assumptions of Theorem 7.4 from [8] the coefficients of the equation can be uniformly estimated on every bounded set with the bound independent of the size of this set.

To apply the above mentioned theorem, we make the following assumption

Assumption 4.1. Let functions $\mu, \mu^{r}, \mu^{H}, \sigma, B, \xi$ and $\Sigma^{-1}$, in addition to being Hölder continuous, be uniformly bounded for all $(t, r), t \in[0, T], r \in \mathbb{R}^{M}$.

Theorem 4.1. Under the assumptions of Theorem 3.1 and Assumption 4.1] there exists a unique solution to the terminal problem (17)-(18). This solution belongs to $H^{1+\beta, 2+\beta / 2}\left([0, T] \times \mathbb{R}^{M}\right)$ and the estimates of Theorem 3.1 hold for $(t, r) \in[0, T] \times$ $\mathbb{R}^{M}$. In particular, the solution is a $C^{1,2}$ function on $[0, T] \times \mathbb{R}^{M}$.

Proof: The proof follows straightforwardly from Theorem 8.1 in Chapter 6 of [8]. That theorem states that the solution to problem (21) is unique and belongs to $H^{1+\beta / 2,2+\beta}\left([0, T] \times \mathbb{R}^{M}\right)$ for some $\beta>0$. It is obvious that such a solution is a function of class $C^{1,2}$.

To use the classical stochastic verification theorem (cf. Theorem 5.1 in Chapter 5 of the book by Yong and Zhou [11]), we have to prove the following simple lemma.

Lemma 4.1. Let $z(t, r)$ be the unique solution to the boundary value problem (17)(18), which exists due to Theorem 4.1] in $[0, T] \times \mathbb{R}^{M}$. Let $q(t, r)=\exp (z(t, r))$ and $\pi^{*}$ be given by equation (13). Then

$$
\begin{aligned}
& \inf _{\pi \in \Pi}\left(\alpha \mu^{X} q+\left(\mu^{r}\right)^{\prime} \nabla_{r} q+\frac{\alpha(\alpha-1)}{2} \theta^{\prime} \theta q+\frac{1}{2} B B^{\prime}\left(\nabla_{r} \otimes \nabla_{r} q\right)+\alpha \theta^{\prime} B^{\prime} \nabla_{r} q\right)= \\
& =\alpha \mu^{*} q+\left(\mu^{r}\right)^{\prime} \nabla_{r} q+\frac{\alpha(\alpha-1)}{2}\left(\theta^{*}\right)^{\prime} \theta^{*} q+\frac{1}{2} B B^{\prime}\left(\nabla_{r} \otimes \nabla_{r} q\right)+\alpha\left(\theta^{*}\right)^{\prime} B^{\prime} \nabla_{r} q,
\end{aligned}
$$

where $\mu^{*}$ denotes the value of $\mu^{X}$, and $\theta^{*}$ the value of $\theta$ evaluated at the point of the optimal strategy $\pi^{*}$.

Proof: The proof is straightforward as the left and right hand sides of the equation in the Lemma are left hand sides of equations (12) and (14), respectively. But equation (14) has been obtained from equation (12) upon substitution (13). The fact that $\pi^{*}$ is an admissible investment strategy has been already stated in Corollary 3.1 From Theorem 4.1 and Lemma 4.1, we easily obtain the verification theorem.

Theorem 4.2. Under assumptions of Theorem 4.1 the function

$$
u(t, x, r)=g(x) q(t, r)
$$

is the value function to the optimization problem (9), where $g(x)$ fulfils the conditions of Assumption 3.1 and $q(t, r)=\exp (z(t, r))$ with $z(t, r)$ being the solution of the boundary value problem (17)-(18), which exists due to Theorem 4.1] 


\section{Conclusions}

In this paper, we have solved the stochastic optimization problem for the loss minimization with the state variable being the ratio of a stochastic benchmark to an investment portfolio. The control parameter of this problem is the portfolio investment strategy. The problem is solved in a market model of $N$ securities being log-normal stochastic processes and depending on $M$ external economic factors. The stochastic benchmark is also a log-normal process but the set of risk factors on which this benchmark depends can be larger than the set of risk factors of the securities making the whole problem an incomplete market problem.

The stochastic optimization problem has been reduced to the HJB equation which, in this case, is a multidimensional quasilinear parabolic equation. Using the general theory of such equations, we have proved that under suitable regularity conditions the HJB equation possesses a unique solution which is sufficiently smooth to guarantee the fulfilment of the stochastic verification theorem. Hence, the solution to the HJB equation is a unique solution to the initial optimization problem. This is a natural extention of similar results obtained in a less general setting without the dependence of security prices on external economic factors.

A natural question which arises is the extension of the obtained results to a market with less restrictive assumpions. The most severe of these assumptions is the boundedness of the coefficients in the whole domain and the lifting of these restrictions will be the subject of future research.

\section{References}

[1] Bouchard, B., Elie, R., Touzi, N.: Stochastic target problems with controlled loss. SIAM J. Control Optim. 48, 3123-3150 (2009)

[2] Bouchard, B., Elie, R., Imbert, C.: Optimal control under stochastic target constraints. SIAM J. Control Optim. 48, 3501-3531 (2009)

[3] Bouchard, B., Vu, T.N.: A stochastic target approach for P\&L matching problems, Preprint Ceremade, University Paris-Dauphine (2011)

[4] Browne, T.: Reaching Goals by a Deadline: Digital options and Continuous-Time Active Portfolio Management. Adv. Appl. Probab. 31, 551-577 (1999)

[5] Browne, T.: - Beating a moving target: optimal portfolio strategies for outperforming a stochastic benchmark. Fin. Stoch. 3, 275-294 (1999)

[6] Dai Pra, P., Runggaldier, W.J., Tolotti, M.: Pathwise optimality for benchmark tracking. IEEE Trans. Automat. Control. 49, 386-395 (2004)

[7] Föllmer, H., Leukert, P.: Quantile hedging. Fin. Stoch. 3, 251-273 (1999)

[8] Ladyzhenskaya, O.A., Solonnikov, V.A., Uraltseva, N.N.: Linear and Quasilinear Equations of Parabolic Type. Amer. Math. Soc. (1968)

[9] Soner, H.M., Touzi, N.: Stochastic target problems, dynamic programming and viscosity solutions. SIAM J. Control Optim. 41, 404-424 (2002)

[10] Soner, H.M., Touzi, N.: Dynamic programming for stochastic target problems and geometric flows. J. Europ. Math. Soc. 4, 201-236 (2002)

[11] Yong, J., Zhou, X.Y.: Stochastic Controls. Hamiltonian Systems and HJB Equations. Springer (1999) 